\title{
Controle da pressão arterial após denervação simpática renal percutânea em paciente com hipertensão arterial resistente
}

\author{
Acadêmicos: Raif Restivo Simão, Marcus Vinicius Rodrigues Agrela, Juliana dos Santos Frassei \\ Orientadores: Thiago Midlej Brito, Pedro Alves Lemos, Luiz Aparecido Bortolotto
}

Introdução: A Hipertensão Arterial Sistêmica (HAS) é uma condição clínica multifatorial e um dos principais fatores de risco para morbidade e mortalidade cardiovascular. A prevalência de HAS no Brasil é cerca de 30\%, sendo que aproximadamente $15 \%$ dos hipertensos não obtêm controle adequado de pressão arterial (PA), apesar do uso de combinações adequadas de anti-hipertensivos. Esse grupo apresenta Hipertensão Arterial Resistente (HAR), definida por PA acima das metas recomendadas $(\geq 140 / 90 \mathrm{mmHg}$ ), em uso de três anti-hipertensivos sinérgicos em doses máximas, sendo um deles diurético; ou quando a PA estiver controlada com uso de quatro ou mais fármacos. Recentemente, tem sido proposto um tratamento intervencionista para HAR, Denervação Simpática Renal (DSR), baseado no bloqueio do estímulo simpático renal eferente e aferente, que parece ter papel fundamental na fisiopatologia da HAS. A DSR, por meio da ablação por radiofrequência $(\mathrm{RF})$, mostrou resultados iniciais muito satisfatórios em estudos internacionais, mas recentemente sua indicação tem sido questionada por não demonstrar benefícios em relação ao tratamento clínico otimizado. No Brasil, há pouca experiência com o procedimento.

Objetivos: Descrever o caso de uma paciente com HAR grave submetida à DSR e, por meio deste, discutir a eficácia da DSR no tratamento da HAR.

Relato do caso: Paciente de 43 anos, do sexo feminino, com história de HAS desde 2007, diagnosticada após quadro de pré-eclâmpsia que acarretou deslocamento precoce de placenta e parto prematuro. Desde então, iniciou crises hipertensivas de repetição, caracterizadas por palpitações, sudorese e cefaleia intensa, com níveis pressóricos de até $290 \times 200 \mathrm{mmHg}$. $\mathrm{Na}$ admissão hospitalar, apresentava PA de 220x100mmHg (sentada) sob uso de 9 anti-hipertensivos e sem outras alterações significativas do exame físico. Apresentava função renal normal, ecocardiograma sem anormalidades e investigação de hipertensão secundária mostrou-se negativa. Foi submetida à DSR por ablação de RF de ambas artérias renais, com cateter irrigado $5 \mathrm{~F}$ conforme protocolo de estudos internacionais, sem intercorrências clínicas.

Cinco meses após o procedimento, a paciente apresentava-se em bom estado geral, assintomática cardiovascular, em uso de três fármacos anti-hipertensivos, com PA =150x90mmHg. Em evolução 18 meses após a DSR, a paciente não apresentou mais crises hipertensivas, assintomática, mantendo PA mais controlada (140x90mmHg) em uso de duas classes de antihipertensivos.

Portanto, em uma paciente com HAR grave, a DSR com cateter de ablação por RF proporcionou melhora significativa no controle da PA, com menor número de medicações, e melhora da qualidade de vida. Assim, a DSR representa uma nova terapia no controle da HAR, atuando em importantes mecanismos fisiopatológicos da HAS, cujos resultados iniciais mostraram respostas satisfatórias no controle das PA dos pacientes com DSR.Apesar da eficácia da DSR ter sido questionada por estudos mais recentes, tem benefício comprovado em pacientes bem selecionados e extremamente refratários à terapia anti-hipertensiva convencional, como demonstrado no relato de caso aqui descrito.

Em conclusão, a DSR é um procedimento seguro e eficaz, se aplicado em casos selecionados de HAR, depois de excluídas causas secundárias de HAS, e que não respondem ao tratamento medicamentoso otimizado e a mudanças de hábitos de vida com adesão plena comprovada. 